\title{
Pulse Dropout in Harmonically Mode-Locked Fiber Lasers
}

\author{
Moshe Horowitz, Curtis R. Menyuk, Fellow, IEEE, Thomas F. Carruthers, and Irl N. Duling, III, Member, IEEE
}

\begin{abstract}
We have studied theoretically and experimentally supermode competition in an actively mode-locked Er-fiber laser that operates in a high harmonic mode. Using an innovative numerical technique that allows us to properly account for the complex interaction of multiple pulses with the gain media, we could study the dynamics that leads to supermode competition, pulse dropouts, and pulse pair generation, and to accurately predict the limits of the stable operating regime.
\end{abstract}

Index Terms-Mode-locked lasers, nonlinear optics, optical fiber lasers, pulsed lasers, solitons.

\section{INTRODUCTION}

A CTIVELY mode-locked Er-doped fiber lasers are potentially important in high-data-rate optical communication systems since they can generate pedestal-free picosecond pulses at a very high repetition rate. These lasers tend to be highly harmonically mode-locked and are therefore potentially subject to supermode competition. As a result, pulse energies in the different time slots may be unequal, corresponding to amplitude fluctuations, dropout, and pulse pair generation.

Analyzing harmonically mode-locked fiber laser systems is a significant theoretical challenge because the laser cavity can contain as many as $10^{4}$ pulses, all of which interact. The direct interaction between pulses is weak in actively mode-locked lasers due to the mode-locker; however, the pulses all indirectly interact via the amplifier. Because of the very slow response time of the Er-doped amplifier, the pulses all affect the amplifier saturation, leading in many cases to highly complex dynamics. Almost all work to date aimed at analyzing these systems studies isolated pulses and assumes that the gain saturation is determined by the energy of the pulse divided by the period between pulses [1]-[3]. In effect, one is assuming that the same pulse is repeated in every time slot. This approach may be used to estimate limits on the minimum and the maximum pulse duration that are needed for stable propagation without dropouts [2], [3]. However, it cannot be used to study the dynamics that leads to

\footnotetext{
Manuscript received August 13, 1999; revised November 24, 1999. This work iwas supported by the U.S. Department of Energy, the National Science Foundation, and by the Wolfson Foundation.

M. Horowitz is with the Department of Electrical Engineering, Technion-Israel Institute of Technology, Haif, 32000, Israel and also with the Department of Computer Science and Electrical Engineering, University of Maryland, Baltimore County, Baltimore, MD 21250 USA.

C. R. Menyuk is with the Department of Computer Science and Electrical Engineering, University of Maryland, Baltimore County, Baltimore, MD 21250 USA, and also with the Laboratory for Telecommunications Sciences, c/o: USARL, Adelphi, MD 20783-1197 USA.

T. F. Carruthers and I. N. Juling, III, are with the Optical Sciences Division, Naval Research Laboratory, Washington, DC 20375-5338 USA.

Publisher Item Identifier S 1041-1135(00)01979-0.
}

supermode competition, pulse dropouts, and pulse pair generation, and therefore it does not accurately predict the limits on the stable operating regime. In principle, one can remove this difficulty by studying a long string of pulses, but in practice it is not feasible even computationally to study long strings, and we have verified that the results slowly converge so that even 24 pulses are not enough to obtain an accurate solution, nor do the results agree well with experiments.

We introduce an innovative yet simple technique that allows us to accurately model the amplifier gain and to efficiently study dynamical effects such as supermode competition. In contrast to previous theoretical work, where the gain was determined only by a limited number of pulses, our approach enables us to accurately calculate the gain saturation due to all the cavity pulses, and we have found out that the gain is one of the most important parameters that determine the performance of the laser and its dynamics. In our simulations, each pulse except one makes its appropriate contribution to the gain dynamics; so, if there are $N$ pulses in the laser cavity, then the contribution of each pulse to the frequency-dependent gain dynamics is weighted by a factor $1 / N$. The one remaining pulse, which we refer to as a superpulse, makes the remainder of the contribution to the gain dynamics and represents the average of all the pulses that are not kept in the computation. This approach converges rapidly as we increase the number of pulses. We believe that this approach will be useful in modeling a wide variety of lasers and storage rings containing many pulses.

In this letter, we apply this approach to modelling supermode competition-particularly pulse dropout—in fiber lasers. We modeled the laser dynamics as it passes through four different operating regimes when the intracavity power increases; three of these regimes were observed experimentally in a dispersion managed sigma laser. Dispersion management makes the last operating regime unobservable both theoretically and experimentally. An excellent quantitative agreement between theory and experiments was obtained.

\section{THEORETICAL MODEL}

The laser configuration in the theoretical model, is similar to that used in the sigma laser [4]. The dispersion map contains $60 \mathrm{~m}$ of fiber with $D_{1}=13.37 \mathrm{ps} / \mathrm{nm} \cdot \mathrm{km}, 26 \mathrm{~m}$ of dispersion compensating fiber with $D_{2}=-92 \mathrm{ps} / \mathrm{nm} \cdot \mathrm{km}, 60 \mathrm{~m}$ of fiber with $D_{3}=13.37 \mathrm{ps} / \mathrm{nm} \cdot \mathrm{km}$, and $46 \mathrm{~m}$ of fiber with $D_{4}=17.7$ $\mathrm{ps} / \mathrm{nm} \cdot \mathrm{km}$. By comparing the theoretical and the experimental results we found out that the average dispersion of this laser $D_{\text {avg }}$ equals $0.14 \mathrm{ps} / \mathrm{nm} \cdot \mathrm{km}$. The modulation frequency was 10 $\mathrm{GHz}$ and the average nonlinear coefficient was $2.1 \mathrm{~W}^{-1} \cdot \mathrm{km}^{-1}$. 
We calculated the pulse propagation in the fiber by solving the nonlinear Schrödinger equation using the split-step Fourier method. The 3-m long erbium-doped fiber amplifier was modeled as an ideal three-level system with a $20-\mathrm{nm}$ bandwidth, a small-signal gain of $22 \mathrm{~dB}$, and a response time of $100 \mu \mathrm{s}$ that does not depend on the intracavity power. We modeled the amplifier saturation by solving the Ginzburg-Landau equation in parallel with the Maxwell-Bloch equations [5] using the split-step Fourier method. Spontaneous emission was simulated as white noise that was was integrated along the whole amplifier. We assume an internal loss of $6 \mathrm{~dB}$ at the mode locker, $1 \mathrm{~dB}$ at each of the two ends of the amplifier, and $2 \mathrm{~dB}$ due to an output coupler located before the amplifier.

Approximately $N=10^{4}$ pulses simultaneously propagate inside the laser cavity. It is only possible to model the propagation of $n=5$ to 24 pulses and we therefore added a superpulse that represents the pulses that are not simulated individually. The cavity energy $E_{t}$ was given by: $E_{t}=W(N-n) E_{s}+\sum_{i=1}^{n} E_{i}$, where $E_{s}$ is the energy of the superpulse and $\sum_{i=1}^{n} E_{i}$ is the sum of the energies of the ordinary pulses in the simulation. The effect of pulse dropout was calculated by including a variable $W$ that equals the fraction of the pulses remaining in the cavity. The value of $W$ was self-consistently determined in our simulation by updating the shape of the superpulse using the ordinary pulses every 2000 iterations, and we checked that the results are not sensitive to this choice. The use of the superpulse guarantees that the saturation energy of the amplifier is not strongly affected by one of the ordinary pulses, so that these pulses can change their shape and even drop out while others equilibrate with the right pulse shape. We have carefully validated this approach by gradually increasing the number of simulated pulses, and we found that the results typically converge beyond $n=5$ pulses. Since the superpulse represents $W(N-n)$ pulses and the response time of the erbium-doped fiber amplifier is slow, the power density of the amplifer noise for the superpulse was taken to be smaller by a factor $[W(N-n)]^{1 / 2}$ relative to the ordinary pulses.

\section{THE Four REgIMES}

When the average power is below $0.5 \mathrm{~mW}$ the nonlinear effect becomes unimportant. Fig. 1(a) shows the numerically calculated pulse train in this first regime; the pulse amplitudes fluctuate independently, leading to supermodes that also fluctuate. This noisy behavior occurs because there is no nonlinear feedback so that pulses can change their amplitudes without affecting their durations [6]. The transition between this regime and the next is caused by the amplified sponataneous emission noise that limits the coherence time of the laser, as we verified by eliminating the noise in our simulation. When the typical nonlinear length becomes shorter than the coherence length of the laser, the laser will operate in the second operating regime.

In the second regime, shown in Fig. 1(b), nonlinear effects become important and decrease the pulse duration from about 4.5 ps to about 3.2 ps. The complex interaction of all the pulses with the gain medium and the mode-locker leads some pulses to drop out and others to stabilize with similar shapes and amplitudes. The dropout occurs since some pulses can decrease their loss in

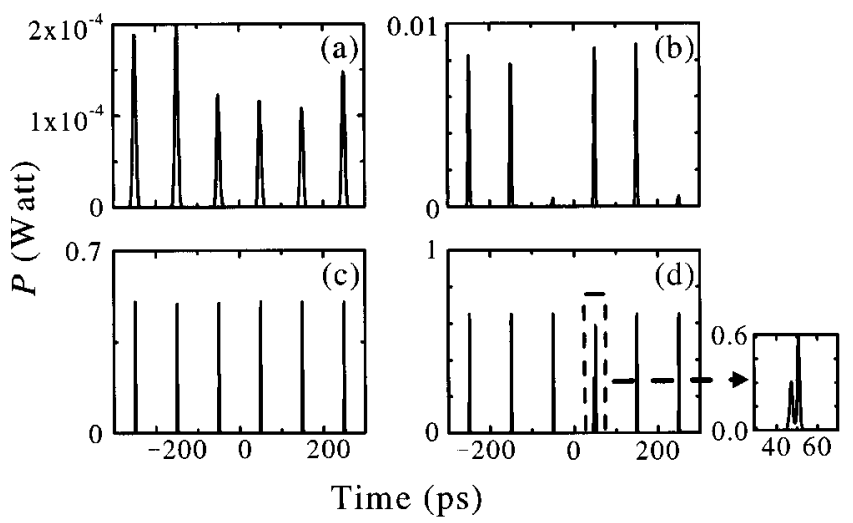

Fig. 1. Pulse train for an output power of (a) $P=14 \mu \mathrm{W}$, (b) $2 \mathrm{~mW}$, (c) 32 $\mathrm{mW}$, obtained for a laser with a sigma configuration. Pulse train for an output power of (d) $P=44 \mathrm{~mW}$ obtained for a laser with a uniform dispersion map with a dispersion coefficient $D=2 \mathrm{ps} / \mathrm{nm} \cdot \mathrm{km}$. Six pulses are simulated and the first pulse is the superpulse. The results displayed here were obtained after 150000 iterations.

the mode-locker by increasing their energy and decreasing their duration due to soliton shortening effect. The increase of the energy of those pulses decreases the amplifier gain due to saturation effects and causes net loss in a roundtrip to other pulses that will eventually drop. As the intracavity power increases, the fraction of the time slots that are filled increases; however, the number of filled slots is not a simple function of the intracavity power. It also depends on the previous state of the laser, since it is possible to remove some fraction of the pulses after they have been established. The others compensate by increasing their energies and decreasing their duration. Therefore, the transition to the next operating regime exhibits a bistable behavior.

Beyond $2.5 \mathrm{~mW}$, we enter the third regime in which all the time slots can be filled, as shown in Fig. 1(c). This power boundary is in good agreement with the power limit calculated using Lyapunov's method for studying the stability of Gaussian pulses assuming that all the pulses have the same amplitude and shape [8]. For hyperbolic-secant pulses that propagate in a cavity with low loss, Lyapunov's method gives the same result as obtained using soliton pertubation theory [2]. When all time slots are filled the equilibrium pulse characteristics may be determined by the conventional analysis [1]; however, including the gain dynamics allows us to calculate the low-frequency noise components due to the amplifier gain, and to analyze dynamical effects, such as the ability of the laser to recover when a pulse is removed due to changes in the enviromental conditions. When the average power $P$ is below $5 \mathrm{~mW}$, some pulses may be removed, as in the second regime; however, above this power level, the system will quickly restore pulses that are removed. Clearly, this regime is the optimal regime in which to operate a laser. By contrast, fiber storage rings which are in many ways analogous to lasers [7], should not work in this regime. The approach described in this paper should allow the user to determine the fraction of pulses that may be safely removed from a storage ring with fixed intracavity power.

In some harmonically mode-locked laser configurations, a fourth operating regime exists in which more than one pulse occupies a single time slot. In the sigma laser we did not observe 
this regime either theoretically or experimentally, because this laser uses a strong dispersion management scheme, making the last regime unobservable. We did, however, observe this regime theoretically in a laser with a uniform dispersion map with a dispersion coefficient of $D=2 \mathrm{ps} / \mathrm{nm} \cdot \mathrm{km}$. When the pulse duration equals $1.4 \mathrm{ps}$ and the laser performance is optimal, the output power in the uniform dispersion laser and in the sigma laser are the same. Fig. 1(d) indicates that when $P>42 \mathrm{~mW}$, some of the time slots develop pulse pairs. The pulse pairs are energetically favourable since they have a narrower bandwidth and lower loss due to the amplifier filtering and nonlinear effects. The energy in a pulse pair is about 1.7 times larger than in a single pulse, and therefore one cannot assume that the energy is equally split between the time slots as assumed in the conventional approach. In previous work, Kärtner et al. [3] found a limit on the minimum pulse duration due to the competition between the pulses and the continuum. In our laser the lower pulse duration is limited by the generation of pulse pair. The difference between the limits occurs, at least in part, because our laser operates in a high harmonic mode so that a small excess of energy in several pulses may accumulate in a single pulse to form a pulse pair rather than a continuum around each pulse.

\section{EXPERIMENTAL RESULTS}

The laser studied experimentally is an actively mode-locked soliton sigma laser [4], which acts much like a polarization-maintaining ring laser. The laser is dispersion-compensated. When the laser is mode-locked at $10 \mathrm{GHz}$, approximately $10^{4}$ pulses circulate in its $192-\mathrm{m}$ cavity. The laser's output as a function of the average cavity power $P$ is presented in Fig. 2 as a series of sampling oscilloscope density contour plots. Three distinct regimes of operation are observed, and the figure presents pairs of plots from near the cavity power boundaries of each regime.

At the lowest optical powers, corresponding to the first regime defined in the previous section, the laser produces noisy pulses with durations at the laser's Kuizenga-Siegman limit [9] of 4.5 ps or longer, as shown in Fig. 2(a). When $P$ rises above $0.5 \mathrm{~mW}$, the laser begins to produce a combination of solitons with 3.3-ps durations [Fig. 2(b)] and dropouts. As $P$ increases the proportion of dropouts decreases and the pulse duration decreases smoothly to $2.6 \mathrm{ps}$.

When $P$ exceeds $5.5 \mathrm{~mW}$ the pulse duration jumps to 3.3 ps and the laser generates an uninterrupted stream of pulses, as Fig. 2(c) demonstrates, corresponding to the third regime. The lower power limit of the stable operating regime is in good agreement with the theoretical minimum power needed for the laser to recover from random pulse dropouts $-5 \mathrm{~mW}$. As $P$ increases to its maximum value of $34 \mathrm{~mW}$, the pulse duration decreases smoothly to $1.4 \mathrm{ps}$ and the production of a dropout-free stream of pulses is maintained. The forces suppressing dropouts are substantial; we have observed pulse dropout ratios below $10^{-14}$ in bit-error-rate measurements with this laser [10]. The amplitude noise becomes smaller as

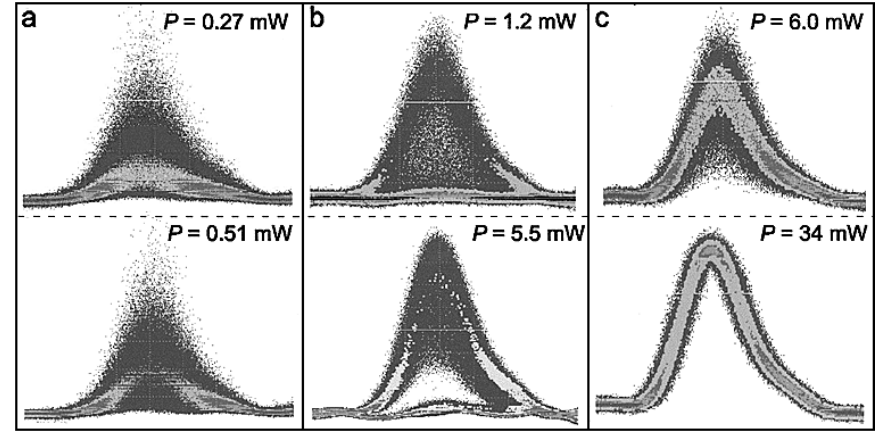

Fig. 2. Sampling oscilloscope density contour plots of the pulses generated by the sigma laser. The three sections of the figure represent the first three operating regimes as a function of the average intracavity power $P$.

$P$ increases, indicating that the available optical power is more uniformly distributed at higher pulse energies.

The fourth regime, that of multiple soliton production in some time slots, was not observed in the sigma laser since the laser cavity is strongly dispersion-managed and, at higher pulse energies, the pulse duration decreases very slowly with incremental increases in the pulse energy [10]-[12]; dropout-free pulse production is observed over a power range of more than a factor of 6 . The role of dispersion management in stabilizing the laser will be addressed in another publication [13].

\section{REFERENCES}

[1] H. A. Haus, "A theory of forced mode locking," IEEE J. Quantum Electron., vol. 11, pp. 323-330, 1975.

[2] D. J. Jones, H. A. Haus, and E. P. Ippen, "Subpicosecond solitons in an actively mode-locked fiber laser," Opt. Lett., vol. 21, pp. 1818-1820, 1996.

[3] F. X. Kärtner, D. Kopf, and U. Keller, "Solitary-pulse stabilization and shortening in actively mode-locked lasers," J. Opt. Soc. Amer. B, vol. 12, pp. 486-496, 1995.

[4] T. F. Carruthers and I. N. Duling, III, "10-GHz, 1.3-ps erbium fiber laser employing soliton pulse shortening," Opt. Lett., vol. 23, pp. 1927-1929, 1996.

[5] M. Horowitz, C. R. Menyuk, and S. Keren, "Modeling the saturation induced by broad-band pulses amplified in an erbium-doped fiber amplifier," IEEE Photon. Technol. Lett., vol. 11, pp. 1235-1237, 1999.

[6] N. Nakazawa, K. Tamura, and E. Yoshida, "Supermode noise suppression in a harmonically modlocked fibre laser by selfphase modulation and spectral filtering," Electron. Lett., vol. 32, pp. 461-463, 1996.

[7] M. F. Nakazawa, E. Yamada, H. Kubota, and K. Suzuki, "10 Gbit/s soliton data transmission over one million kilometers," Electron. Lett., vol. 27, pp. 1270-1272, 1991.

[8] M. Horowitz and C. R. Menyuk, "Analysis of pulse dropout in harmonically modelocked fiber lasers using Lyapunov's method," Opt. Lett., vol. 25, pp. 40-42, 2000.

[9] D. J. Kuizenga and A. Siegman, "FM and AM mode locking of the homogeneous laser-Part I: Theory," IEEE J. Quantum Electron., vol. 6, pp. 694-706, 1970.

[10] T. F. Carruthers, M. L. Dennis, I. N. Duling, III, M. Horowitz, and C. R. Menyuk, "Enhanced stability of a dispersion-managed, harmonically mode-locked fiber laser," in OSA Tech. Dig., Conf. Lasers and ElectroOptics. Washington, DC, 1999, pp. 101-102.

[11] N. J. Smith, N. J. Doran, F. M. Knox, and W. Forysiak, "Energy-scaling characteristics of solitons in strongly dispersion-managed fibers," Opt. Lett., vol. 21, pp. 1981-1983, 1996.

[12] T. Yu, E. A. Golovchenko, A. N. Pilipetskii, and C. R. Menyuk, "Dispersion-managed soliton interactions in optical fibers," Opt. Lett., vol. 22, pp. 793-795, 1997.

[13] T. F. Carruthers, I. N. Duling, III, M. Horowitz, and C. R. Menyuk, "Dispersion management in a harmonically mode-locked fiber soliton laser," Opt. Lett., to be published. 